\section{Germline edits: heat does not help debate}

Edward Lanphier and colleagues marshal a familiar and, we believe, ultimately counterproductive line of argument against genetically modifying the human germline (Nature 519, 410-411;2015, see also Nature http://doi.org/3xt; 2015).

In his essay The Rhetoric of Reaction (Belknap, 1991), the late economist Albert Hirschman describes three simplifying, stereotypical theses that are often invoked to preserve prevailing policies or practices. Lanphier et al. follow these same welltravelled tracks. They warn that a public outcry over the ethics of germline editing could hinder therapeutic applications of nonheritable genetic modification of somatic cells (the perversity thesis); that the technique might not deliver the anticipated benefits (the futility thesis); and that modifying genes in reproductive cells could be a slippery slope towards nontherapeutic genetic enhancement (the jeopardy thesis).

Such blinkered arguments can lead to sharply polarized views, obstructing the balanced and pragmatic societal discussion and careful studies that should precede possible applications of human germline modification (see D. Baltimore et al. Science 348, 36-38; 2015).

Mika Martikainen, Ole Pedersen Newcastle University, UK. mika.martikainen@newcastle.ac.uk

\section{Germline edits: trust ethics review process}

Edward Lanphier and colleagues contend that human germline editing is an unethical technology because it could have unpredictable effects on future generations. In our view, such misgivings do not justify their proposed moratorium (Nature 519, 410-411;2015).

When in vitro fertilization and preimplantation genetic diagnosis were first introduced, they had unpredictable consequences. Both went on to transform reproductive medicine.

Many nations already have ethics review processes that assess the risks of experiments on human embryos, with the prospect of even stricter evaluation standards as new fertility techniques come along (I. G. Cohen et al. Science 348, 178-180; 2015).

There is no reason to close off whole avenues of controversial research when they have barely begun (see, for example, Nature http://doi.org/3xt; 2015). Germline editing is a revolutionary technology that potentially offers an enormous range of benefits to the next generation.

Julian Savulescu, Chris Gyngell, Tom Douglas University of Oxford, UK. julian.savulescu@philosophy. ox.ac.uk

\section{Lift sanctions now to save public health}

The international accord on Iran's nuclear programme agreed this month in Switzerland offers no timeline for lifting international economic sanctions, which profoundly affect public health (see, for example, S. Shahabi et al. Nature 520, 157; 2015). This must be urgently rectified because, as the Iranian health minister has stressed, it will take a year to restore public-health systems after sanctions are lifted.

By driving up prices and limiting the availability of medicines, sanctions are forcing people and clinics to use poor-quality, black-market medications. A shocking example still under investigation is last month's unexpected permanent loss of vision in 15 people after eye surgery in a Tehran clinic, with unknown numbers similarly affected at two other centres. The tragedy is thought to have arisen from a non-standard ampoule (see go.nature.com/oyudtj; in Persian).

Health care is a fundamental human right (see go.nature. com/xqtarv and go.nature. $\mathrm{com} / \mathrm{xuoeyb}$ ). Specialists in human rights, such as the United Nations special rapporteur on human rights in Iran, Ahmed Shaheed, should remind the US Congress and other Western governments of the importance of lifting sanctions and of a clear strategy to rapidly improve the country's public health.

Mehdi Aloosh Montreal, Canada. Arash Aloosh BI Norwegian Business School, Oslo, Norway. md_aloosh@hotmail.com

\section{Double-blind peer review a double risk}

Double-blind peer review of research papers is a worthy idea but has two flaws in practice (Nature 518, 274; 2015).

First, most modern research builds on previous studies published by a limited number of groups. This makes it almost impossible to write a paper without revealing with near certainty who the authors are, even if they manage to avoid such giveaways as "we showed previously that... (No Longer Anonymous et al., 2012)".

The second flaw is more serious. To function in our increasingly competitive research culture, in which misconduct is on the rise, researchers need to be aware of which labs can be trusted and which have a record of irreproducibility. If a highly regarded lab and one with a questionable reputation each submit reports of similar investigations, a good reviewer would be extra vigilant in assessing the less-reliable lab's study, even though the same evaluation standards would be upheld for both.

Double-blind peer review removes this crucial qualitycontrol option, opening the way for mediocre and bad labs to clutter the literature with sub-standard science.

Thomas E. DeCoursey Rush

University Medical Center,

Chicago, Illinois, USA.

tdecours@rush.edu

\section{Better policing for fishy catch data}

Missing catch data are a big problem in achieving sustainability of world fisheries (Nature 519, 280-282; 2015).

The accuracy of catch data is also important — particularly for endangered fish species.

Take the common skate (Dipturus batis), now classified as critically endangered in the Red List of the International Union for Conservation of Nature. In 2009, the European Union put a total ban on fishing for this species and made it illegal to retain these fish on vessels, exchange them between boats, or land them.

We were therefore surprised to learn that official landing records of D. batis in 2014 by UK vessels in the United Kingdom and Europe, and by foreign vessels in UK ports, showed commercial catches totalling 1.8 tonnes. In 2011-13, the total recorded catch was even higher at 7.8 tonnes (all data by written request from the UK Marine Management Organisation).

The reliability of these figures is unclear, however, given that catch totals are compiled from assorted data collected at numerous UK ports. Local inaccuracies might be perpetrated through systematic errors in species identification, for example, or by mistakes in the codes allocated at market.

It is essential for the future of vulnerable species that catch data are properly checked by the authorities to monitor adherence to bans, and that punitive action is taken against contraventions. David W. Sims, Samantha J. Simpson Marine Biological Association of the United Kingdom, Plymouth, UK. dws@mba.ac.uk 